\title{
i \\ Multispectral Focal Plane Assembly for Satellite Remote Sensing
}

\author{
Jeffrey Rienstra, \\ Sandia National Laboratories \\ P.O. Box 5800, M/S 0972 \\ Albuquerque, NM 87185-0972 \\ 505-845-8682 \\ jlriens@sandia.gov \\ Mary Ballard, Santa Barbara Research Center
Goleta, CA 93117
805-562-2729
mballard@ccgate.hac.com \\ 75 Coromar Dr., Bldg B3, M/S 85
}
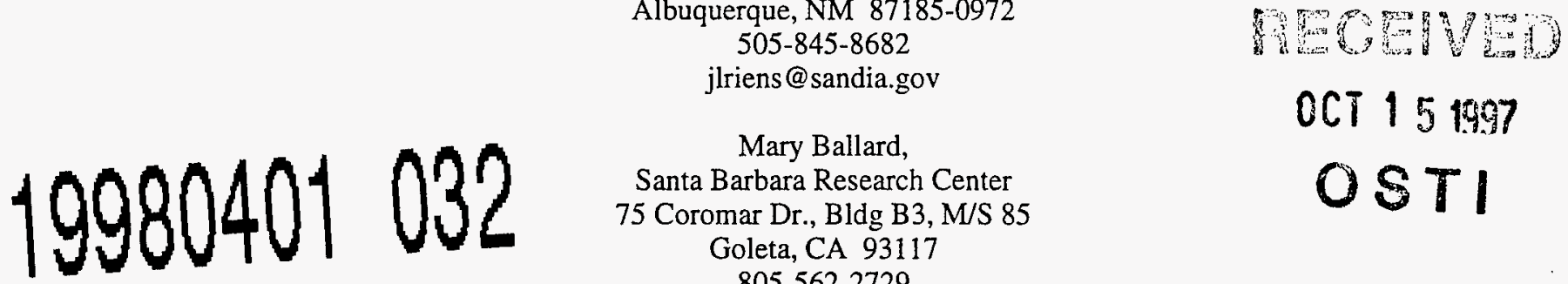

\begin{abstract}
Sandia National Laboratories and several subsystem contractors are developing technologies applicable to multispectral remote sensing from space. A proof of concept multispectral sensor system is under development. The objective of building this sensor is to demonstrate and evaluate multispectral imaging technologies for various applications. The three major subsystems making up the sensor are the focal plane assembly (FPA), the cryocooler, and the telescope. This paper covers the focal plane assembly, which is the basis of the sensor system. The focal plane assembly includes sensor chip assemblies, optical filters, and a vacuum enclosure with cold shielding. Linear detector arrays provide spatial resolution in the cross-track direction for a pushbroom imager configuration. The optical filters define 15 spectral bands in a range from $0.45 \mu \mathrm{m}$ to $10.7 \mu \mathrm{m}$. All the detector arrays are mounted on a single focal plane and are designed to operate at $75 \mathrm{~K}$. No beam splitters are used. The four spectral bands covering the visible to near infrared have roughly 2400 pixels each, and the remaining 11 spectral bands have roughly 600 pixels each. The average total rate of multispectral data from the FPA is approximately 15.4 megapixels per second.
\end{abstract}

There are several challenges encountered when laying out these diverse spectral bands on a single focal plane. First of all, each spectral band uses three staggered arrays to span the required length, because the cross-track dimension of the focal plane (approximately $30 \mathrm{~mm}$ ) is too great for a single detector array made from $\mathrm{InSb}$ or $\mathrm{HgCdTe}$. The tolerance for array-to-array height differences is very tight because of the small depth of focus of the telescope. Secondly, all the detectors must operate at $75 \mathrm{~K}$. Silicon photodiodes with unit cell preamplifiers are used to achieve high performance at the focal plane operating temperature and to minimize cross-talk. Finally, the entire focal plane packaging approach must minimize the thermal load to the cryocooler. The multispectral focal plane assembly includes a cold shield with an aperture positioned at the exit pupil of the telescope to minimize the thermal background from tie uncooled telescope structure. All of these considerarions make this a challenging space sensor to design and build.

At the time this paper is being written, the multispartral focal plane assembly is in the fabrication phase. A thermal/mechanical mockup has been built and tested for tia vibration environment and to determine the thermal loaj. Some of the sensor chip assemblies and filters have ben built and tested. Several notable features of the design arz covered in the paper as well as preliminary test data.

\section{TABLE OF CONTENTS}
1. INTRODUCTION
2. SENSOR DESCRIPTION
3. FPA DESCRIPTION
4. FPA PERFORMANCE
5. CONCLUSIONS

\section{INTRODUCTION}

The purpose of this paper is to describe a multispectral focal plane assembly for satellite remote sensing. Multispactral remote sensing from space has been provided for quite some time by a number of existing systems. Examples are Landsat Thematic Mapper (TM)[1], Advanced Very High Resolution Radiometer (AVHRR)[2], and SPOT[3]. These multispectral imaging systems provide a wealth of userul information relating to earth resources and environmenial monitoring. TM and AVHRR cover the range of wavelengths from the visible to long wavelength infrared but use scanning optics to form an image and beam splitters to 
DISCLAIMER

This report was prepared as an account of work sponsored by an agency of the United States Government. Neither the United States Government nor any agency thereof, nor any of their employees, makes any warranty, express or implied, or assumes any legal liability or responsibility for the accuracy, completeness, or usefulness of any information, apparatus, product, or process disclosed, or represents that its use would not infringe privately owned rights. Reference herein to any specific commercial product, process, or service by trade name, trademark, manufacturer, or otherwise does not necessarily constitute or imply its endorsement, recommendation, or favoring by the United States Government or any agency thereof. The views and opinions of authors expressed herein do not necessarily state or reflect those of the United States Government or any agency thereof. 
divide the spectral content onto several focal planes. SPOT is a pushbroom imager with long, linear arrays, but it only covers visible wavelengths and also uses beam splitters with several focal planes. In this paper we describe an advanced focal plane assembly that embodies a unique, straightforward, and simple design for multispectral imaging over a broad range of wavelengths. Our approach is based on a single focal plane with 15 spectral bands in a pushbroom imager.

The next section of this paper is a general description of the overall sensor system and short description of the subsystems. Section three contains a more detailed description of the focal plane assembly (FPA) and its major components, including the readout integrated circuits. Following that is a section identifying the most significant FPA performance parameters and preliminary test data for some of them. The last section is a summary.

\section{SENSOR DESCRIPTION}

\section{General}

The objective of building this research and development multispectral sensor is to demonstrate and evaluate advanced multispectral imaging technologies for satellite remote sensing. The sensor system consists of the telescope, focal plane, cryogenic cooler, and system electronics, making up a multispectral, pushbroom type imaging system. Linear detector arrays with optical filters immediately above the arrays are placed at the focal plane of the telescope. The linear arrays provide spatial resolution in one direction (cross-track). The motion of the sensor relative to the ground produces a scanning operation in the other direction (along-track), thereby producing two-dimensional images in multiple wavelength bands. For this prototype system the multispectral images are stored in memory and retrieved later for archiving and processing.

Table 1 shows the designations for the 15 bands of the multispectral sensor system. All the bands lie between 0.45 $\mu \mathrm{m}$ and $10.7 \mu \mathrm{m}$. The first four are usually referred to as the visible (VIS) bands. The next three bands are the near infrared (NIR). Following that are three short wavelength infrared (SWIR) bands and two medium wavelength infrared (MWIR) bands. The last three bands are the long wavelength infrared (LWIR) bands. Individual bands are referred to by letter, $A$ through $O$. Band $O$ was added after the other bands were identified, which explains why it is out of sequence.

This sensor incorporates a single focal plane design; no beam splitters are used. This is in contrast with the other sensor systems mentioned above. All of them use beam splitters and more than one physical focal plane to include all the spectral bands. All detectors are maintained at the same temperature, nominally $75 \mathrm{~K}$, with a long-life cryogenic cooler.
One of the overriding considerations in the design of this sensor system is radiometric accuracy. It will undergo extensive laboratory calibration. In addition, a built-in calibration system will be used to monitor performance of the imaging system and to correct for drift in detector output.

\begin{tabular}{|c|c|c|}
\hline Band & $\begin{array}{c}\text { Wavelength range } \\
(\mu \mathrm{m})\end{array}$ & Characteristics \\
\hline \hline A & $0.45-0.52$ & VIS (blue) \\
\hline B & $0.52-0.60$ & VIS (green) \\
\hline C & $0.62-0.68$ & VIS (red) \\
\hline D & $0.76-0.86$ & VIR (deep red) \\
\hline E & $0.86-0.89$ & NIR \\
\hline F & $0.91-0.97$ & NIR \\
\hline G & $0.99-1.04$ & NIR \\
\hline H & $1.36-1.39$ & SWIR \\
\hline I & $1.55-1.75$ & SWIR \\
\hline O & $2.08-2.35$ & SWIR \\
\hline$J$ & $3.50-4.10$ & MWIR \\
\hline K & $4.87-5.07$ & MWIR \\
\hline L & $8.00-8.40$ & LWIR \\
\hline M & $8.40-8.85$ & LWIR \\
\hline N & $10.2-10.7$ & LWIR \\
\hline
\end{tabular}

Table 1. Prototype sensor spectral bands.

\section{Telescope}

The telescope for the R \& D multispectral sensor system is a three-mirror anastigmatic design. The unobscured telescope design provides aperture diffraction limited performance in the LWIR bands and very respectable performance in the shorter wavelength bands as well. The mirrors are uncooled, and the telescope cavity is maintained at approximately $275 \mathrm{~K}$. The telescope design provides an exit pupil which is accessible and allows very effective cold shielding of the warm optical structure.

\section{On-Board Calibration System}

The telescope includes several subassemblies that make up the on-board calibration system. The aperture door is double hinged to allow two different surfaces to fill the telescope field of view to provide end-to-end calibration of the imaging system. In the door closed position, the aperture door assembly constitutes a temperature-controlled 
blackbody radiator for calibrating the infrared detector channels. In the door open position, a diffuse reflective panel can be opened up to allow reflected sunlight to illuminate the visible and short wavelength detectors. In addition, there is a quick calibration wheel inside the telescope near the exit pupil. A visible wavelength lamp and two blackbody sources at different temperatures can be placed in view of the detectors within seconds of an imaging sequence to mitigate the effects of detector drift. One of the wheel positions is a retroreflector to provide a low level infrared signal and to reduce the radiative heat load from the warm telescope cavity onto the focal plane assembly.

\section{Cryocooler}

The cryocooler provides over 2.25 watts of cooling power at $65 \mathrm{~K}$. A thermal strap connects the cold block of the cryocooler to a pedestal of the focal plane assembly that supports the detector arrays. The cryocooler also cools the cold shield to reduce the infrared background on the focal plane. The cryocooler uses temperature sensors to provide closed loop temperature control of the cold block. It is designed for low vibration to minimize jitter of the imaging system.

\section{Electronics}

The electronics subsystem performs a number of functions related to the focal plane assembly, the telescope, and the cryocooler. The functions relative to focal plane image data include amplification and digitization of serial data from the focal plane readout circuits, image compression, data storage, and data encoding. In addition, the electronics subsystem provides timing and control signals to the focal plane readout circuits. High resolution temperature control of the focal plane is also provided by this subsystem.

\section{FPA DESCRIPTION}

\section{General Description}

Focal Plane - The cross-track dimension of the focal plane is nearly $30 \mathrm{~mm}$. It would be extremely difficult with today's technology to build MWIR or LWIR detector arrays to cover the entire cross-track dimension with a single linear array. Therefore, three staggered linear arrays are used for each spectral band. The visible wavelength through midwavelength infrared bands are grouped on one type of sensor chip assembly (SCA). The long wavelength infrared bands are grouped on a different type of SCA. One VIS/MWIR SCA is paired with one LWIR SCA to cover approximately one third of the cross-track dimension; three staggered SCA pairs are used to span the full cross-track field of view. The arrangement of the SCAs on the focal plane is shown in Figure 1.

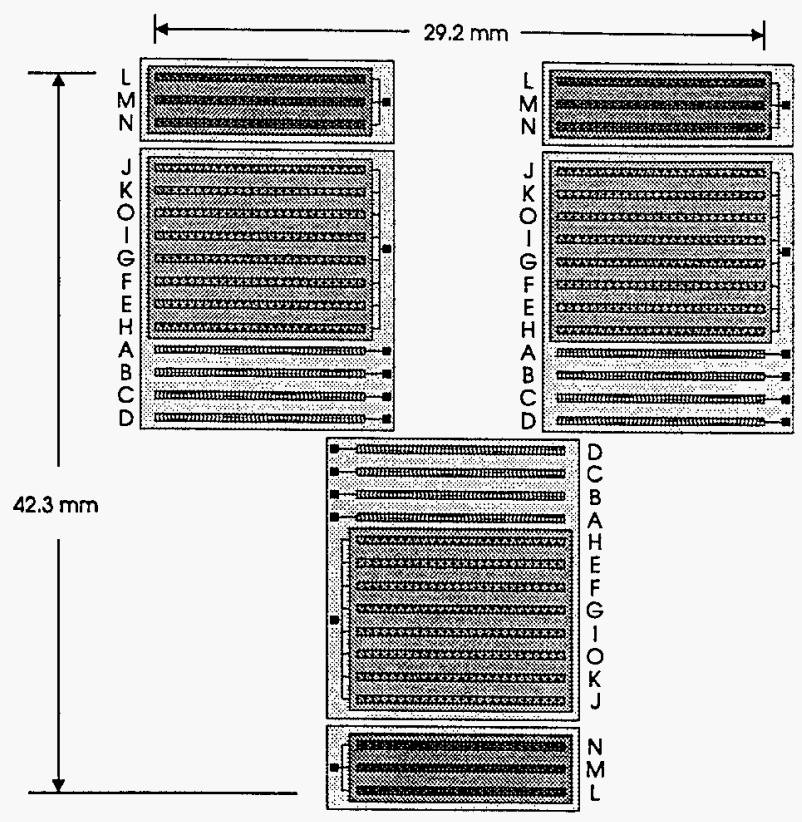

Figure 1. Focal plane layout.

Three different detector materials are used for the various wavelengths. Silicon photodiodes are used for bands A-D. Indium antimonide is used for bands $\mathrm{E}-\mathrm{K}$ and $\mathrm{O}$. Mercury cadmium telluride is used for bands L-N. The order of the bands on the SCAs are also shown in Figure 1. The particular order of bands was chosen to keep the detector arrays made of the same material grouped together and to meet image quality requirements.

These SCAs use hybrid focal plane array technology[4,5]. wherein an array of backside-illuminated photodetectors is attached with cold-welded indium interconnects to a readout integrated circuit (ROIC). The ROIC has a capacitivefeedback transimpedance amplifier corresponding to each pixel which amplifies and stores the signal from the detector. Multiplexing circuitry on the ROIC produces a serial output of values which can be digitized, stored, and processed.

Mechanical Configuration-Figure 2 shows a cutaway view of the focal plane assembly which illustrates the mechanical configuration of the unit. The three SCAs pairs are mounted on individual motherboards which have electrical traces to conduct control waveforms and analog signals between the SCAs and the cables. The cryogenic cables complete the connection between the cold focal plane and the cable connectors attached to the warm part of the assembly. A filter assembly positions the cold band-pass filters directly above the appropriate detector arrays. The cold shield minimizes the infrared background on the detector arrays and maintains a stable operating environment. Finally, the vacuum enclosure surrounds the focal plane components and allows the SCAs and filters to be cooled during laboratory testing.

One of the principal challenges of the this design is to maintain the relative height of the individual SCAs with very tight tolerances. The extremely short depth of focus of the telescope demands that the height variation be less than \pm 15 
$\mu \mathrm{m}$ for the VIS bands and less than $\pm 50 \mu \mathrm{m}$ for the LWIR bands.

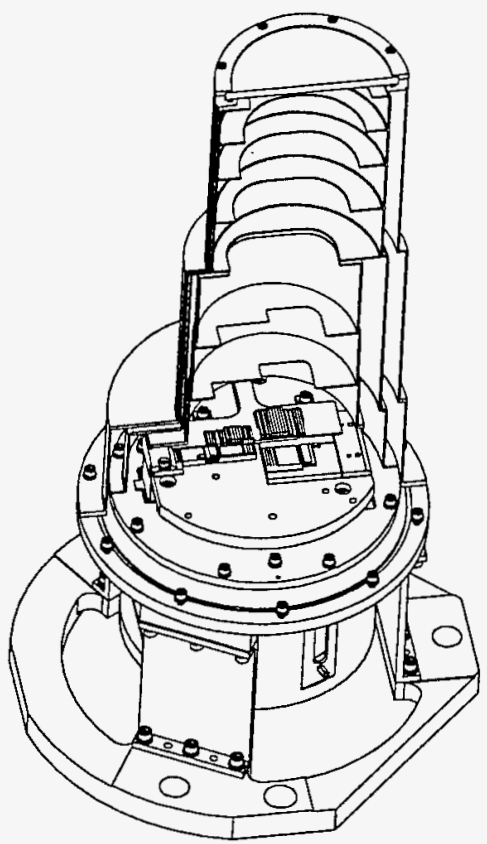

Figure 2. Cutaway view of focal plane assembly.

\section{FPA Components}

Detector Arrays-The detector arrays for bands A-D are formed in the same silicon chip that makes up the ROIC for the InSb detectors. There are 828 photodiode detectors per SCA for each of the four visible bands. Photodiodes were chosen to ensure high performance at the operating temperature of $75 \mathrm{~K}$. The pixels are $12.5 \mu \mathrm{m}$ square and are laid out in two staggered rows separated by $12.5 \mu \mathrm{m}$. The layout of the silicon photodiode detectors is shown in Figure 3(a). A guard ring surrounds each individual pixel, reducing the cross-talk between pixels and enhancing spatial resolution. The active detector area is defined by apertures in an opaque coating that is applied to the silicon surface. This opaque coating shields the amplifiers and multiplexing circuitry from incident light.

Bands $\mathrm{E}-\mathrm{K}$ and $\mathrm{O}$ utilize InSb photodiode detectors. These bands are fabricated on a single piece of InSb which is hybridized to a silicon chip which contains the readout circuitry for these bands as well as the detectors and readouts for bands A-D. There are 207 detectors per SCA for each of the bands $E-K$ and $O$. The pixels for these bands are $50 \mu \mathrm{m}$ square. The layout of the InSB detectors is shown in Figure 3(b). Two rows of detectors are fabricated for each of the InSb bands. This provides a primary and an alternate pixel, individually selectable. For all the InSb bands except $\mathrm{H}$, pixel selection circuitry in the ROIC is used to connect either the primary or the alternate detector to the readout, thereby increasing array operability. Both rows of band $\mathrm{H}$ detectors are read out so they can be combined later to improve the signal-to-noise ratio.

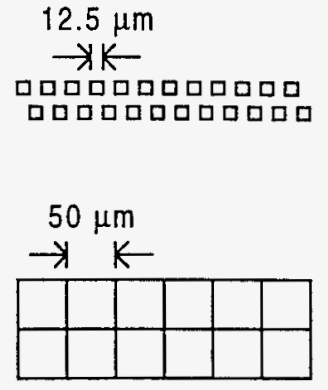

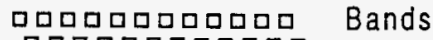 ロロロロロロロロロロロ A.D

(a)

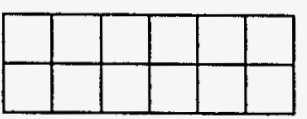

Bands

E-O

Figure 3. Detector array layout.

The detector arrays for bands $\mathrm{L}-\mathrm{N}$ are made from $\mathrm{HgCdTe}$. These bands also have 207 pixels per SCA, with $50 \mu \mathrm{m}$ square pixels. Like the InSb detectors, two rows of photodiodes are made for each band and pixel selection is used to increase operability. These bands utilize a separate ROIC chip from the other bands because of yield considerations.

Readout Integrated Circuits-The ROICs for all the bands are based on a common amplifier design for the unit cells. A capacitive-feedback transimpedance amplifier (CTIA)[6] is used to integrate photocurrent from the corresponding detector and convert the electrical charge into a voltage which can be multiplexed with the signals from other detectors and read out. This circuit design provides very low noise and very high linearity.

A simplified schematic of the unit cell amplifier is shown in Figure 4. This shows the CTIA which integrates photocurrent during each frame. The switch controlled by the clock, $\phi_{\mathrm{rst}}$, resets the integrator. A band limiting capacitor reduces the noise bandwidth. The switch controlled by $\phi_{\mathrm{cl}}$ works in conjunction with $\phi_{\mathrm{rst}}$ to level shift the signal. Following a buffer amplifier, the signal is transferred to the sample and hold capacitor by $\phi_{\mathrm{sh}}$. This allows all the pixels be reset simultaneously and the signal corresponding to each pixel to be read out sequentially during the next integration period. A second buffer amplifier is used for driving the signal out of each unit cell onto the signal bus when it is accessed by $\phi_{\mathrm{en}}$. Not shown is the output amplifier on the output video line which drives the serial data stream off chip.

The total data rate from the focal plane is determined by the number of pixels and the frame time. The nominal frame time for bands A-D and L-N is $715 \mu$ s, and the nominal frame time the other bands is $2.86 \mathrm{~ms}$. There are a total of 2,484 pixels in each of bands A-D and 621 pixels in each of the other bands. Including the fact that both the primary and alternate pixels for band $\mathrm{H}$ are read out, the total data rate for the focal plane is approximately 15.4 megapixels per second.

Even though a common circuit design is used for all the spectral bands, some tailoring is required due to different background signal levels expected for each band and 
different sensitivity requirements. These differing requirements can be fulfilled for the most part by changing the value of the feedback capacitor in the unit cell. A larger capacitor provides for higher irradiance levels without saturating. A smaller capacitor provides a larger conversion gain from photocurrent to output voltage.

One of the features of the ROIC design is a variable integration time. The clock waveforms that activate the reset, clamp, and sample switches determine what fraction of the total frame time is used for integrating photocurrent and leakage current. This value can be changed during operation of the sensor in accordance with operating experience and the expected irradiance levels for a particular imaging sequence. Variable integration time essentially becomes a variable gain setting which can be used to make the most of the available dynamic range of the ROIC.

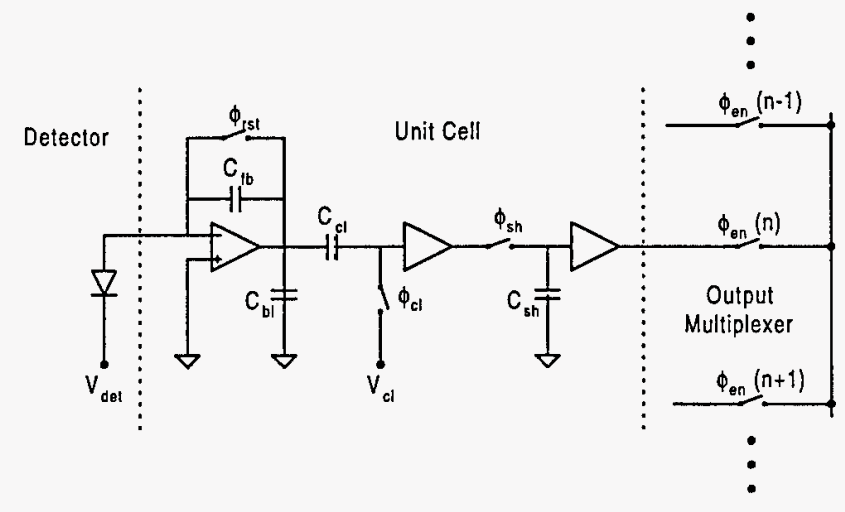

Figure 4. Unit cell schematic.

Filter Assembly-The function of the filter assembly is to position optical filters over the detector arrays to define the appropriate spectral bands. The filters are attached to the focal plane and operate at nearly the same temperature as the focal plane to reduce the infrared background.

The filters must be mechanically fixed above the detector arrays. Groups of filters are bonded together, side-by-side into filter assemblies. The VIS-MWIR bands make up one group and the LWIR bands are the other. These filter assemblies are bonded to a metal bezel which is attached to the focal plane pedestal. Figure 5 shows the arrangement by representing a cross-section of the focal plane in the alongtrack direction.

The optical filters must pass the desired range of wavelengths and reflect or absorb wavelengths outside that range. To maintain radiometric accuracy the out-of-band blocking must be extremely effective. The filters use multilayer dielectric films to define the pass bands. Other layers are added to form low-pass or high-pass blocking layers. A composite filter substrate is used for some bands which includes a layer of absorbing glass.

The thickness of each filter is tailored to equalize the focus for each spectral band. Due to the different materials used for the filter substrates and the change in index of refraction with wavelength, the total focus shift introduced by these materials is different. The thickness of each filter substrate is calculated so that the focus for each band will occur at the appropriate place.

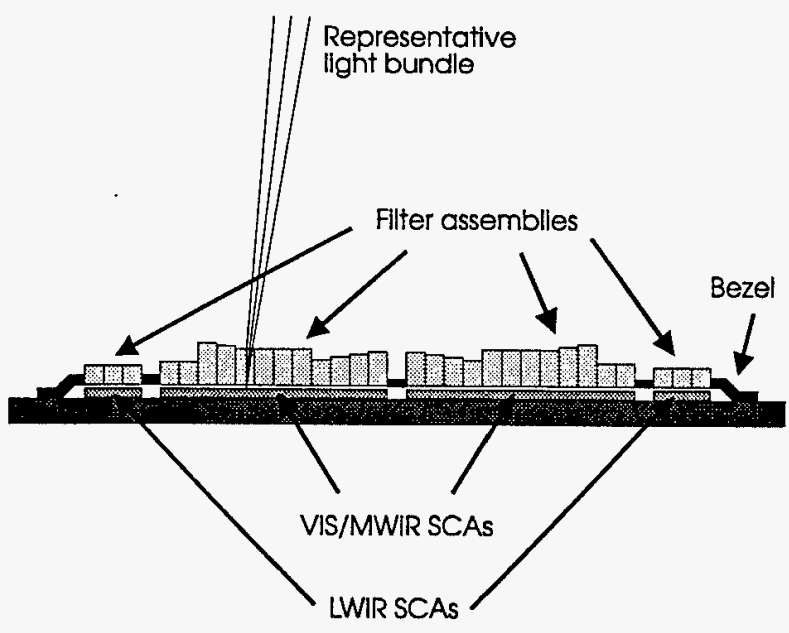

Figure 5. Filter/bezel assembly.

Motherboard/Cryogenic Cables-A separate motherboard is used for each SCA pair. One VIS/MWIR and one LWIR SCA are attached to a motherboard which in turn is attached to the focal plane pedestal. Wire bonds are used to connect the SCAs to electrical traces on the motherboard. At the edge of the pedestal are cryogenic cables which are wirebonded to the motherboard traces and carry the electrical signals from the cold focal plane region to the relatively warm vacuum enclosure. These cables must have good electrical conductivity but low thermal conductivity to minimize the thermal load of the focal plane assembly on the cryocooler.

Cold Shield - The cold shield performs two major functions. The first is to limit the field of view of the warm telescope structure from the focal plane, reducing the background irradiance on the SCAs, especially in the LWIR bands. The cold shield surrounds the SCAs and is in good thermal contact with the cold focal plane. It extends out to the exit pupil of the telescope and defines the aperture of the system. The cold shield is surrounded by a low emissivity radiation shield which reduces the thermal load on the cryocooler by shielding the coldest parts of the focal plane assembly from the warm walls of the dewar.

The second function is to reduce the amount of stray light that reaches the focal plane. The cold shield includes internal baffles to impede stray light reflections from reaching the focal plane. The dimensions and spacing of the baffles as well as the highly absorbing surface finish are designed to minimize stray light.

Dewar Assembly-The dewar assembly puts together all the components listed above into a single unit and provides a vacuum enclosure so the focal plane assembly can be tested in the laboratory. The dewar assembly also acts as the principal interface between the telescope and the focal plane 
assembly. The dewar assembly includes a barium fluoride window which is transparent to the entire range of wavelengths for this sensor. Electrical connectors are provided as well as a vacuum pump-out port. The dewar assembly serves as the precision interface between the telescope and the detectors on the focal plane and accurately locates the detectors at the best focus of the optical system.

\section{FPA PERFORMANCE}

\section{Predicted Performance}

Detectors - The starting point for determining focal plane radiometric performance is the detectors. A common figure of merit for infrared detector performance is RoA, the product of detector dynamic resistance at zero bias and the detector area. This quantity is a strong function of temperature. The minimum RoA product at $75 \mathrm{~K}$ necessary to meet the focal plane performance requirements is $1 \times 10^{5}$ $\mathrm{ohm}-\mathrm{cm}^{2}$ for the InSb detectors and $95 \mathrm{ohm}-\mathrm{cm}^{2}$ for the $\mathrm{HgCdTe}$ detectors.

Readouts - The readout integrated circuits also affect the sensitivity of the focal plane assembly. Very low noise is desired but must be balanced with the requirement for very large charge handling capacity for some of the spectral bands. The CTIA circuits in the unit cell provide excellent noise performance and very good dynamic range. One way to gauge the performance of a ROIC is to determine the number of noise electrons per pixel per frame. Table 2 shows the predicted number of noise electrons for each band.

Quantum Efficiency-The detector quantum efficiency (QE) is the measure of the response of the detector to incoming photons. The detectors used for this sensor system have relatively high quantum efficiency. Predicted $\mathrm{QE}$ for each band is also shown in Table 2. Achieving uniformly high quantum efficiency requires high quality starting material for the detectors and well-controlled detector fabrication processes.

Filter Transmission-The optical filter transmission characteristics are tailored for each band. The two main considerations are in-band transmission and out-of-band rejection. Very good out-of-band rejection is required to perform precise spectral radiometry. In-band transmission must be high enough to meet the detector sensitivity goals. The predicted average in-band filter transmission for each band is shown in Table 2 .

Noise Equivalent Irradiance-The noise equivalent irradiance (NEI) is the overall sensitivity parameter used to evaluate the performance of the detectors on the focal plane. NEI includes the effects of detector RoA, quantum efficiency, readout noise, and filter transmission. The NEI requirement for each band was chosen to provide adequate signal-to-noise ratio to perform the desired radiometric measurements. Table 2 shows the predicted NEI performance for each band. For most bands the predicted NEI is well below the requirement.

One important consideration for making accurate radiometric measurements is the linearity of response of the detectors and readout circuits. The detectors will be calibrated at a number of irradiance levels in the laboratory and during their useful lifetime. The response of each pixel should be very nearly linear with the incident irradiation. As it turns out, there will be integral nonlinearity of a few percent over the range of irradiance expected. However, the nonlinearity for each pixel is quite stable. The deviation from linearity can be mapped out during laboratory calibration and data that are collected can be corrected.

\begin{tabular}{|c|c|c|c|c|}
\hline Band & $\begin{array}{c}\text { Read noise } \\
\text { (electrons) }\end{array}$ & $\begin{array}{c}\text { QE } \\
(\%)\end{array}$ & $\begin{array}{c}\text { Filter } \\
\text { transmission } \\
(\%)\end{array}$ & $\begin{array}{c}\text { NEI } \\
\left(\mathrm{ph} / \mathrm{cm}^{2} \text {-sec }\right)\end{array}$ \\
\hline \hline A & 53 & 68 & 76 & $2.5 \times 10^{11}$ \\
\hline B & 60 & 81 & 81 & $2.2 \times 10^{11}$ \\
\hline C & 57 & 75 & 86 & $2.1 \times 10^{11}$ \\
\hline D & 58 & 65 & 76 & $2.8 \times 10^{11}$ \\
\hline E & 205 & 85 & 76 & $2.0 \times 10^{10}$ \\
\hline F & 432 & 85 & 78 & $2.1 \times 10^{10}$ \\
\hline G & 213 & 85 & 76 & $1.8 \times 10^{10}$ \\
\hline H & 93 & 85 & 50 & $3.0 \times 10^{9}$ \\
\hline I & 463 & 85 & 71 & $3.4 \times 10^{10}$ \\
\hline O & 209 & 85 & 71 & $1.7 \times 10^{10}$ \\
\hline J & 391 & 85 & 76 & $1.9 \times 10^{10}$ \\
\hline K & 227 & 85 & 76 & $1.7 \times 10^{10}$ \\
\hline L & 1880 & 65 & 81 & $3.2 \times 10^{11}$ \\
\hline M & 2130 & 65 & 81 & $3.7 \times 10^{11}$ \\
\hline N & 2270 & 65 & 81 & $5.3 \times 10^{11}$ \\
\hline
\end{tabular}

Note: Integration time is $700 \mu \mathrm{sec}$ for bands A, B, C, D, L, $\mathrm{M}$, and $\mathrm{N} ; 2850 \mu \mathrm{sec}$ for all other bands

Table 2. Predicted performance for focal plane.

Thermal Load-A focal plane performance item of great concern is the thermal load that the focal plane assembly places on the cryocooler. Part of the thermal load is due to the electrical power dissipation in the detectors and readout integrated circuits. The largest part of the thermal load comes from radiative and conductive interactions of the focal plane assembly with the surrounding telescope structure which is uncooled. The total thermal load is within the budget determined by the capability of the cryocooler and the desired design margin. 
Filters-Filter assemblies have been fabricated and spectral transmission measurements have been made. Figures 6-9 show the measured transmission curves for each of the bands. Although some of the filters do not quite meet the predicted values for average in-band transmission, the overall focal plane sensitivity is still expected to meet requirements. In general, the filter spectral characteristics are quite good.

Due to the optical system design, the angle of incidence to the filters varies across the focal plane. The spectral characteristics of interference filters depends on angle of incidence, so there are slightly different spectral characteristics at different focal plane locations within each band. These variations have been modeled and evaluated.

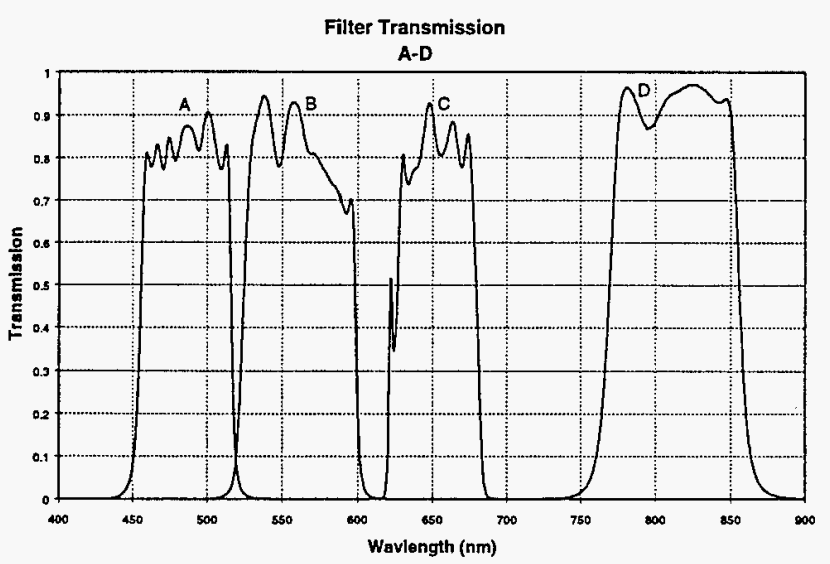

Figure 6. Filter transmission, bands A-D.

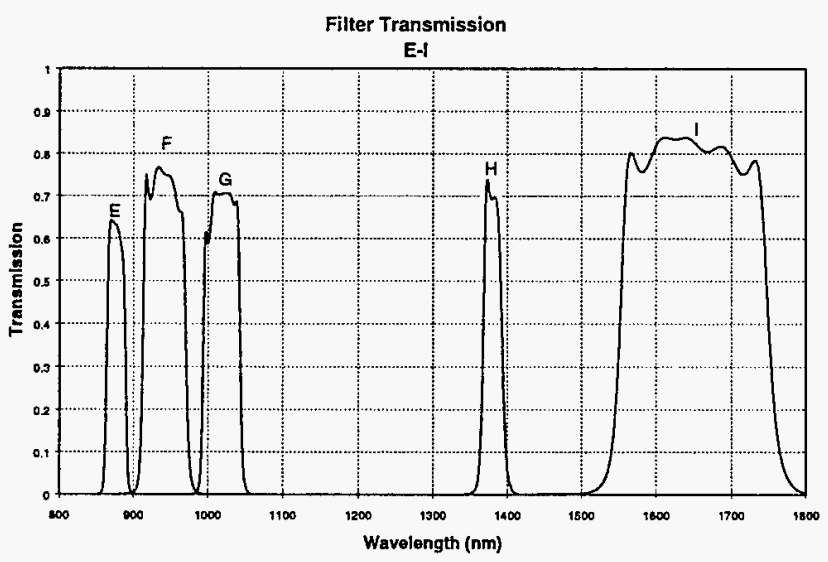

Figure 7. Filter transmission, bands E-I.

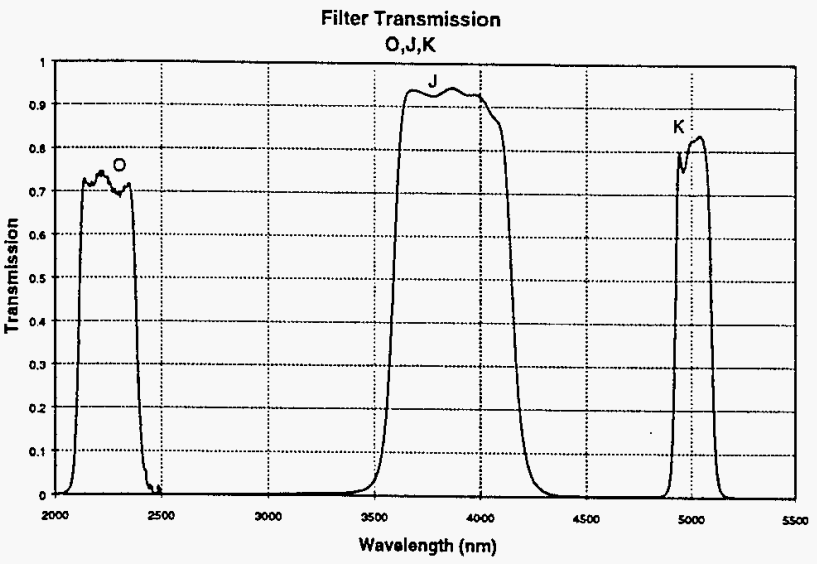

Figure 8. Filter transmission, bands $\mathrm{O}, \mathrm{J}$, and $\mathrm{K}$.

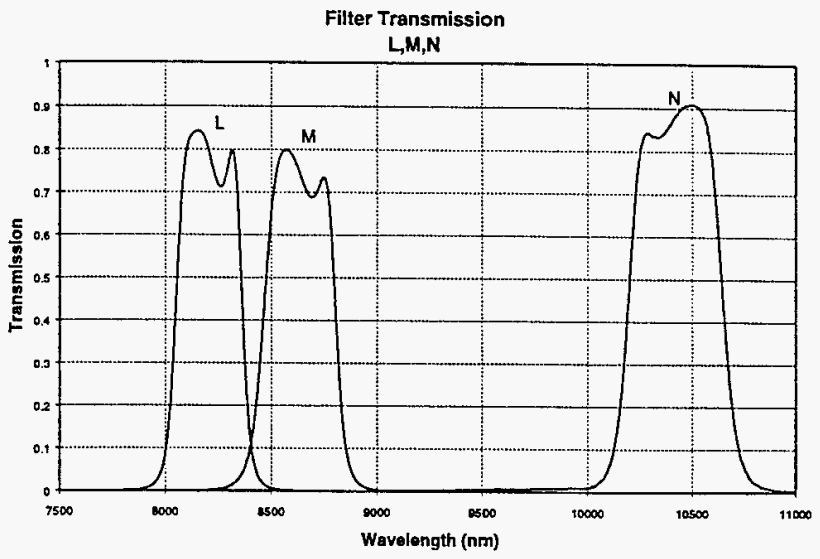

Figure 9. Filter transmission, bands L, M, and N.

Sensor Chip Assemblies-A number of LWIR SCAs have been tested and the flight units have been identified. Figure 10 shows the measured responsivity for all six linear arrays (primary and alternate pixels for bands $\mathrm{L}, \mathrm{M}$, and $\mathrm{N}$ ) of the LWIR SCA number 66674). These measurements are performed without the spectral bandpass filters. The average responsivity is different for each band because each one has a different feedback capacitor value. The uniformity within each band is quite good, with only one or two dead pixels within each. Because of the pixel select circuitry on the ROIC, the dead pixels can be switched out and replaced with the alternate pixels.

A histogram of raw responsivity for the primary pixels of band $\mathrm{N}$ is shown in Figure 11. If the two dead pixels from this band shown in Figure 10 are replaced with their respective alternates, the resulting uniformity is $1.78 \%$. This is very good for LWIR HgCdTe detectors.

The measured noise for all six linear arrays on the same LWIR SCA at $80 \mathrm{~K}$ is shown in Figure 12. There is not the same distinction among the bands as was shown in the responsivity data, and there is greater spread in the data. The average noise level is a bit higher than expected in the 
sensor system, because these measurements were taken about $5 \mathrm{~K}$ higher than the nominal operating temperature.

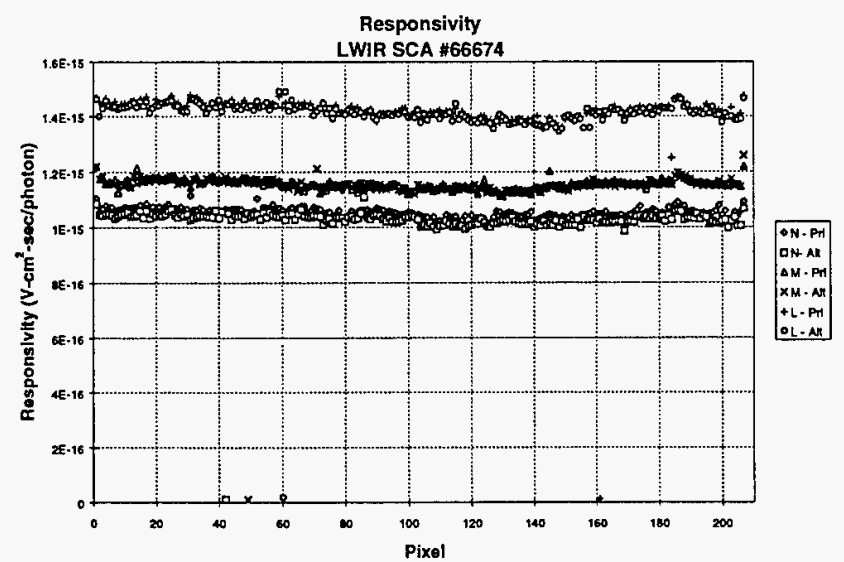

Figure 10. SCA responsivity.

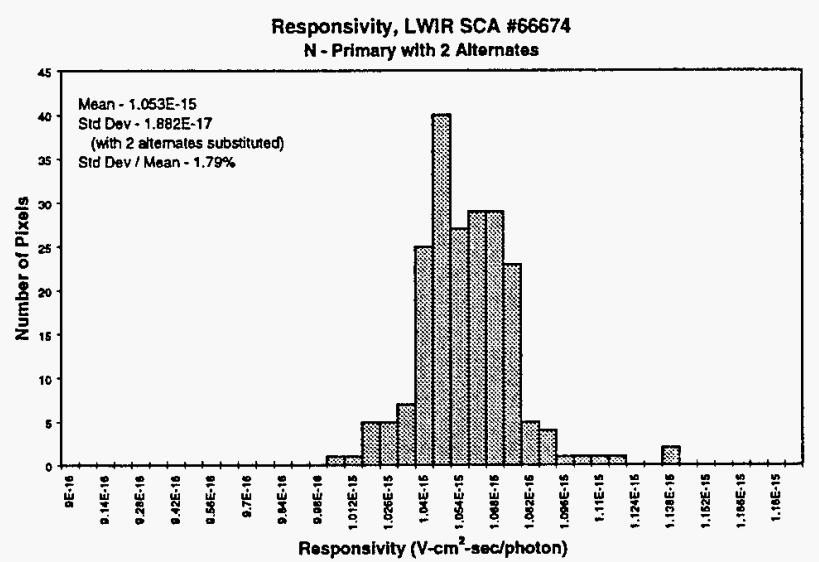

Figure 11. Histogram of SCA responsivity.

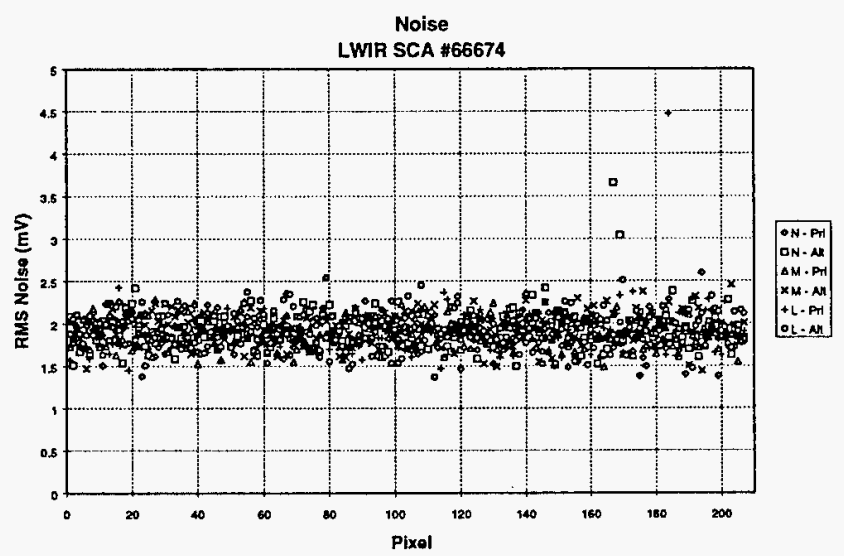

Figure 12. SCA noise.

Finally, the NEI calculated from responsivity and noise for LWIR SCA number 66674 is shown in Figure 13. Average NEI is higher than the predicted value shown in Table 2. As mentioned above, these tests were taken at a higher temperature than the normal operating temperature. The measured values, though higher than the goal, are well below the required NEI. These data are representative of the high quality LWIR detector arrays that have been produced for this program.

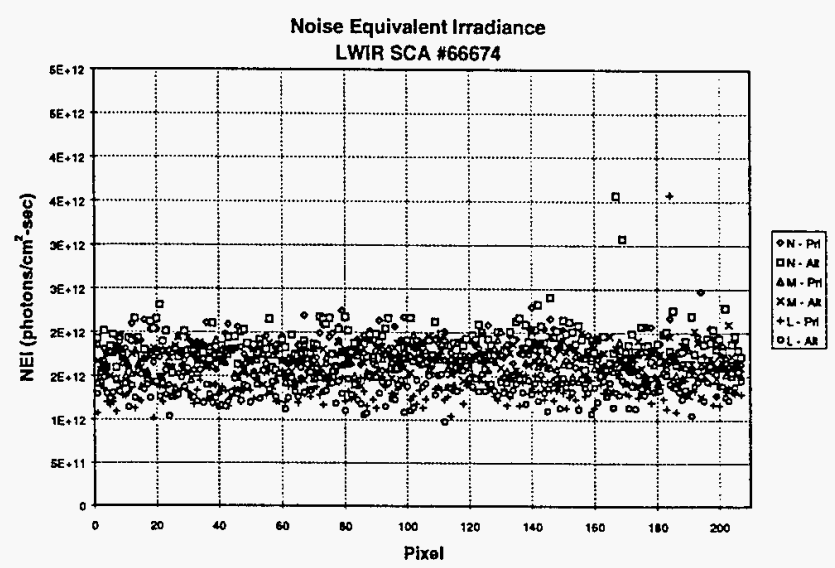

Figure 13. SCA noise equivalent irradiance.

The VIS/MWIR SCAs have not yet been through acceptance testing, but preliminary screening tests show that they will also meet our expectations. Integration of the flight SCAs into the dewar assembly will then proceed.

\section{CONCLUSIONS}

This focal plane assembly embodies an approach to challenging technical requirements that combines proven detector and readout circuit technologies with innovative operational and packaging techniques. The overview of the sensor system provides a context for appreciating the key performance aspects of the focal plane assembly. The focal plane approach that has been selected is expected to produce a final product that meets all design requirements and has potential for other multispectral imaging applications.

\section{REFERENCES}

[1] C. B. Pease, Satellite Imaging Instruments, Chapter 14, Chichester, England: Ellis Horwood Limited, 1991.

[2] C. B. Pease, Satellite Imaging Instruments, Chapter 12, Chichester, England: Ellis Horwood Limited, 1991.

[3] C. B. Pease, Satellite Imaging Instruments, Chapter 16, Chichester, England: Ellis Horwood Limited, 1991.

[4] M. R. Kruer, D. A. Scribner, and J. M. Killiany, "Infrared focal plane array technology development for Navy applications", Optical Engineering 26, 182-190, March 1987. 
[5] Joseph T. Longo, Derek T. Cheung, A. Michael Andrews, C. C. Wang, and John M. Tracy, "Infrared Focal Planes in Intrinsic Semiconductors", IEEE Transactions on Electron Devices ED-25, 213-232, February 1978.

[6] John L. Vampola, "Readout Electronics for Infrared Sensors", Volume 3, Chapter 5, The Infrared \& ElectroOptical Systems Handbook, Ann Arbor, Michigan: Environmental Research Institute of Michigan, 1993.

Jeff Rienstra is a Senior Member Technical Staff at Sandia National Laboratories and works on satellite sensor systems. He is responsible for design, analysis, procurement, and performance characterization of photodetectors and focal plane arrays for satellite payloads. While at the Air Force Weapons Laboratory he investigated radiation effects on infrared focal plane arrays and microelectronics. He obtained a B.S. degree in physics from the University of North Dakota in 1977 and earned an M.S. degree in physics from the Massachusetts Institute of Technology in 1984.

Mary Ballard is a Focal Plane Array Systems Engineer at Santa Barbara Research Center and leads a technical team to build and deliver focal plane / dewar assemblies. She is responsible for initial design concept, specification flowdown, and performance analysis of space qualified focal plane hardware. She has also developed test plans and characterized the radiation hardness of space sensor components. She obtained a B.S. degree in electrical engineering from the University of California at Santa Barbara in 1988. 
M98000387

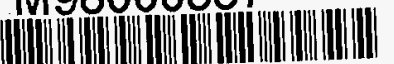

Report Number (14) SAND-97-2481C

Jubl. Date (11) 1997.10

sponsor Code (18) DOE/NN, XF

JC Category (19) UC-900, DOE/ER

DOE 